\title{
Understanding life science majors' ideas about diffusion
}

\author{
Samuel Luke Tunstall, ${ }^{1}$ Abhilash Nair, ${ }^{2}$ Kathleen Hinko, ${ }^{2,3}$ Paul Irving, ${ }^{2}$ and Vashti Sawtelle ${ }^{2,3}$ \\ ${ }^{1}$ Mathematics Education, Michigan State University, 220 Trowbridge Rd, East Lansing, MI USA 48824 \\ ${ }^{2}$ Department of Physics \& Astronomy, Michigan State University, 567 Wilson Rd, East Lansing, MI USA 48824 \\ ${ }^{3}$ Lyman Briggs College, Michigan State University, 919 E. Shaw Ln, East Lansing, MI USA 48824
}

Diffusion and osmosis are central concepts in biology that explain mechanisms of passive transport. While biology education research has documented significant student struggles with these concepts, there are very few studies from chemistry and physics education research that explore student understanding of these ideas. Diffusion is a concept that cuts across disciplines and has important underlying physics principles that contribute to students' understanding of the process (e.g., a series of collisions resulting in a random motion process). In an introductory physics for life science majors course we documented the initial ideas students have about the topic of diffusion. We then designed a unit that leverages computational simulations to develop student understanding of how elastic collisions underlie diffusive motion. We present findings from this preliminary study on student resources for understanding diffusion, and discuss the implications for revising the curricular materials as a result of our work.

\section{INTRODUCTION AND BACKGROUND}

The concept of diffusion is one that involves knowledge from multiple disciplines and represents an important topic in multiple science classes [1]. To date, however, the work exploring how students understand and learn diffusion has been primarily limited to research in biology education [2]. From a physics education perspective, only a single paper has explored ideas about diffusion, and that paper did not include any student data [3]. Though it is not inherently problematic that extant research on students' understanding of discussion is primarily grounded in biology education, because diffusion involves knowledge from multiple disciplines, there is merit in exploring the topic from another discipline's context. In this study we present preliminary data on the knowledge about diffusion that students in an Introductory Physics for Life Science Majors class bring into the course with them. Using this information, we designed a unit focusing on computational simulations that builds on the knowledge students bring to the course. Our aim is to describe students' incoming resources in this study, the impact of such on our computation unit design, and the implications of this work for future research and teaching.

\section{THEORETICAL FRAMEWORK}

The biology education literature primarily addresses diffusion from the perspective of identifying and eliminating misconceptions (see for example, $[4,5]$ ). The problem with this perspective (that of misconceptions, not of biology) is that it tends to assume that students' beginning ideas are unproductive and need to be replaced by conceptually correct ideas. We instead approach the question of student ideas about diffusion from a resources perspective [6], specifically using the notion of resource as a way of thinking about a construct, idea, or process-in this case, diffusion. This perspective allows us to focus our instructional design on modifying the resources students activate and to consider what additional conceptual resources students might need to be activated. In this study we bring a resources framework to understanding the knowledge that students bring into class with them. We acknowledge that students develop ideas from diffusion outside of physics classrooms, and thus in our work we leverage the resources framework to explore the range of conceptual ideas students bring to this topic.

\section{METHOD}

The context for this exploratory study is an Introductory Physics for Life Science Majors course. The course was designed to bridge content from physics and biology [7]. We collected data across three sections of this course (with two instructors) for a total of 124 students enrolled. Instructional material on the topic of diffusion was introduced early in the semester (week 5) by building from the topics of conservation of momentum and energy. The computational activities on diffusion were embedded in this week of the material. To explore students' resources for understanding diffusion at the beginning of the semester, the instructors of the course asked all students to respond to a discussion prompt in the first week of the course. Instructors used the prompt as a low-stakes homework grade and placed it on the university's online learning management system. Of the 124 students enrolled in the course, 119 responded. The prompt was:

This week in class we are going to focus primarily on modeling diffusion through simulations. In this discussion post I would like you to put together a definition of diffusion. You may use whatever resources are helpful to you, but I will note that different disciplines will define this term differently.

From the responses, the first author crafted an emergent coding scheme for resources; the author team then met as a group to discuss the emergent scheme and to aggregate 
them into a manageable list. As with any coding process, it is important to note that categorization masks some of the nuance in students' responses; not every student's response that we marked in the "high-to-low" category, for example, used that exact phrase. Moreover, a limitation of the data arises from the medium itself, in that students could read others' responses and respond to those posts rather than writing their own unique post. After creating and solidifying the coding scheme for student resources, the first author coded students' responses; the team then met again to discuss the full coding, picking several responses randomly as a means of spot-checking the first author's work. In analyzing the data, the first finding was that the majority of students used several resources in their responses (see Table I). Then, in order to explore how students used these resources in conjunction with one another, we analyzed the connections between the resources in student responses. To do so we used an odds ratios [8] and associated confidence intervals (after checking that the assumptions were met). We determined the likelihood that-within the dataset—students who employed one resource also employed another resource. From this work came our second finding. Using our exploration of students incoming resources for defining diffusion we then designed an instructional unit that built from these resources. In the final section of this paper we describe this unit and an initial evaluations of the implementation.

\section{INCOMING RESOURCE ANALYSIS}

A significant result from the coding of students' discussion forum responses is that the vast majority of students came in with conceptions of diffusion tied to their course experiences in biology and chemistry. This finding makes sense in that physics is typically taken by the students after these other two courses at our university. Table I shows the percentages of students whose responses fell into each resource category. To interpret the categories, note that any given category should be interpreted as students having said that diffusion relates to, involves, or applies to that category.

Notably, most students (84\%) described diffusion as the movement of things from a high area of concentration to one with low concentration. We emphasize things to convey the rich diversity of ways students described what was moving; for some, it was the movement of particles or molecules in an unstated space, while for others it was the movement of particles within cells. For others yet, it was the movement of Febreze particles in a room, or crowds of people at a concert.

An example of a student's response that was grounded in a biological context is below:

Diffusion is the movement of particles from high concentration to low concentration. Osmosis uses diffusion and a semipermeable membrane to create solvents of equal concentrations. Osmotic pressure is the pressure that prevents the
TABLE I. Pre-semester diffusion conceptions.

\begin{tabular}{cl}
\hline \hline Conception of Diffusion & $\begin{array}{l}\text { Percentage of Students } \\
(n=119)\end{array}$ \\
\hline High-to-low concentration & $84 \%$ \\
Relates to energy & $55 \%$ \\
Balance, homeostasis, or equilibrium & $50 \%$ \\
Spreading or dispersing of particles & $39 \%$ \\
Dynamic & $30 \%$ \\
Randomness & $27 \%$ \\
Concentration gradient & $16 \%$ \\
Collisions & $11 \%$ \\
Spreading of energy or momentum & $10 \%$ \\
Entropy & $8 \%$ \\
\hline \hline
\end{tabular}

solvent from moving across the membrane that wouldn't be in equal concentrations ... [High-tolow concentration]

Not only does this student use resources from biology to descibe what diffusion is, but they also use mechanistic reasoning to explain how it happens. Causal reasoning - though not directly requested in the prompt, or coded for in this analysis-was common in many students' responses.

In addition to the clear influence of previous courses on students' discussion of high-to-low, related remnants of prior courses are also seen in the resources of balance (or homeostasis or equilibrium), and energy (manifesting most as passive or active transport), as seen in the examples below.

I have taken many science classes and I define diffusion in a more biological sense rather than physics sense (if there is one). When I think of diffusion I think of a process in which a substance spreads from high concentration gradient to low concentration gradient without the use of ATP. It is a passive process that can be coupled with active transport pumps, such as the Na-K pump. Diffusion is used to get cells basic nutrients such as $\mathrm{O} 2$ and N2. It is also used in big processes involving neurons in the body. [Relates to energy]

In a solution, the concentration of a solute will favor a state in which it is uniform. In order to accomplish this, molecules will move from an area of high concentration to low concentration. When no energy is required to do this it is referred to as simple diffusion. A special category of diffusion is osmosis, the diffusion of water across a barrier. [Balance, homeostasis, or equilibrium]

Another result was that few students discussed collisions or the spreading of energy or momentum in their description 
of diffusion. This is an interesting finding, given that many of these students had previously taken an introductory chemistry course where collisions and interactions between particles are emphasized [9, 10]. This is also an interesting finding as these represent a set of resources that an introductory physics course might expect to develop. As Table I suggests, and highlighted explicitly in Table II, most students used more than one resource in describing diffusion.

TABLE II. Discussion forum number of resources.

\begin{tabular}{cl}
\hline \hline Number of resources & $\begin{array}{l}\text { Percentage of students } \\
(n=119)\end{array}$ \\
\hline 0 & $1 \%$ \\
1 & $6 \%$ \\
2 & $22 \%$ \\
3 & $31 \%$ \\
4 & $17 \%$ \\
5 & $17 \%$ \\
6 & $6 \%$ \\
\hline \hline
\end{tabular}

Notably, most students noted at least two resources, and the majority $(71 \%)$ had at least three resources they articulated in their response. Of course, the number of resources students used is not necessarily indicative of the complexity or correctness of their response; rather, it gives us an indication more broadly of how many resources for understanding diffusion students initially activated.

\section{A. Links between resources}

As discussed in the Methods section, we also utilized odds ratios-which are measures of association between two events - to understand statistical links between specific resources within the dataset. An odds ratio of 1 or lower for two resources $a$ and $b$ indicates that $a$ has a likelihood that is not mathematically affected by the presence of resource $b$. An odds ratio greater than 1 indicates that $a$ is more likely to occur in the presence of $b$ than when $b$ is not present. Confidence intervals at the $90 \%$ level resulted in meaningful odds ratio intervals when the interval was outside of one. Of the 81 pairings of resources to form odds ratios, 11 were meaningful, 6 of which are in Table III. The 6 included in Table III were chosen for their importance (in our view); other significant pairings, such as that between high-to-low and the spreading of particles, were of less interest, given that we would expect them to be associated with one another.

Analysis of the confidence intervals for the odds ratios yielded a few noteworthy relationships. To begin, the 100 students who used "high-to-low" as a resource were significantly less likely to have also discussed collisions. Furthermore, those whose responses included the spreading out of things other than particles, as well as those who described the
TABLE III. Odds ratio and confidence intervals.

\begin{tabular}{cl}
\hline \hline $\begin{array}{c}\text { Resource pair }(\boldsymbol{a} ; \boldsymbol{b}) \\
\text { read as “ } \boldsymbol{a} \text { given } \boldsymbol{b} \text { ” }\end{array}$ & $\begin{array}{l}\text { Confidence interval } \\
\text { for odds ratio }\end{array}$ \\
\hline Relates to energy; spreading of particles & $0.33-1.00$ \\
Entropy; relates to energy & $0.02-0.65$ \\
Randomness; dynamic & $4.59-23.30$ \\
Collisions; spreading of energy/momentum & $2.00-16.70$ \\
Collisions; high-to-low & $0.10-0.72$ \\
Collisions; dynamic & $1.42-10.30$ \\
\hline \hline
\end{tabular}

process as dynamic, were more likely to have included collisions in their response. We highlight these statistical connections because they center on concepts attended to in the course and that-as discussed below-instructors used in the designing of an instructional unit in the course.

\section{IDENTIFYING STUDENT RESOURCES POST-INSTRUCTION}

In our Introductory Physics for Life Science Majors course our goal is to design a unit that builds from the resources students have for reasoning about diffusion discussed in the previous sections. Our curriculum was designed to encourage students to activate resources from other course contexts, though did not specifically build from the resources identified in the previous section. The unit on diffusion, which lasted about a week during the semester, started from PERbased curricular resources to develop students understanding of conservation of momentum and energy [11], and then built up a series of VPython computational programs.

Diffusion was used as a guiding context throughout the semester and was a topic revisited on the final exam. Students responded to a question concerning hypothetical students' discussion of a computational unit on diffusion. The question required students to make connections between differing approaches to talking about diffusion (e.g., if Student A discussed diffusion using a computational model, how does that discussion relate to Student B whose discussion centered on entropy?). Specifically, the question was the following (note that the code accompanying the question is not included in this paper):

Context: Suppose that three students are studying for this exam and are discussing the topic of diffusion. Three statements of theirs are below, followed by questions:

Bob: I know from previous classes that diffusion is just the process of particles moving from an area of high concentration to a region of low concentration.

Alice: In the computational problems we did in class, diffusion was just the result of conservation of momentum. 
Paul: In the last unit of the course we discussed how diffusion is the result of increasing entropy in the system.

Questions: How did Alice use the code to decide that diffusion was linked to collisions? How can you connect what Alice and Bob are saying? How can you connect what Alice and Bob are saying to Paul's statement?

Following the same open-coding approach as with the initial discussion prompt, we analyzed students' responses to the question and coded for emergent themes, building upon the previous resource list and adding new resources (not seen in Table I) as necessary. In Table IV below, we present the results from the coding, not including resources that were directly primed in the question's wording itself. We include these results here not for the sake of attributing causality of the unit in (perhaps) shifting students' initial resources, but rather to highlight the additional resources for reasoning about diffusion elicited by the final exam question shown above.

TABLE IV. Final exam diffusion resources.

\begin{tabular}{cl}
\hline \hline Conception of Diffusion & $\begin{array}{l}\text { Percentage of Students } \\
(n=123)\end{array}$ \\
\hline Spreading or dispersing of particles & $70 \%$ \\
Randomness & $51 \%$ \\
Microstates & $37 \%$ \\
Order/disorder & $35 \%$ \\
Velocity & $34 \%$ \\
Balance, homeostasis, or equilibrium & $20 \%$ \\
Concentration gradient & $3 \%$ \\
High-to-low concentration & $2 \%$ \\
\hline \hline
\end{tabular}

[1] J. Shen, O. L. Liu, and S. Sung, "Designing interdisciplinary assessments in sciences for college students: An example on osmosis," International Journal of Science Education 36, 17731793 (2014).

[2] B. W. Dreyfus, B. D. Geller, D. E. Meltzer, and V. Sawtelle, "Resource letter TTSM-1: Teaching thermodynamics and statistical mechanics in introductory physics, chemistry, and biology," American Journal of Physics 83, 5-21 (2014).

[3] E. M. Kramer and D. R. Myers, "Five popular misconceptions about osmosis," American Journal of Physics 80, 694-699 (2012).

[4] E. A. Marek, C. C. Cowan, and A. M. Cavallo, "Students' misconceptions about diffusion: How can they be eliminated?" The American Biology Teacher 56, 74-77 (1994).

[5] A. L. Odom and L. H. Barrow, "High school biology students' knowledge and certainty about diffusion and osmosis concepts," School science and mathematics 107, 94-101 (2007).

[6] D. Hammer, "Student resources for learning introductory physics," American Journal of Physics 68, S52-S59 (2000).
In Table IV, we find about half of the students discussed randomness, and one-third discussed microstates or order/disorder-essential resources fostered in the instructional unit. Only two students (or 2\%) mentioned high-to-low in their final responses.

The analysis of this final exam question suggests that students wrote about diffusion differently at the end of the semester than they did in their initial discussion post. This is a positive sign that the course is supporting students to develop additional resources in understanding diffusion. Our analysis of the initial incoming resources provides a strong first step in understanding how to better leverage students' initial resources for diffusion. For example, in future iterations of the course we hope to build from the high-to-low concentration resource to support students building connections with ideas of randomness. Moreover, these findings are an important step toward designing more targeted questions for the coming year for the discussion forum, mid-semester exams, and final exam, among other data collection opportunities. The goal of designing more targeted questions is not to see students' changes over the course of the semester, but to see how instruction might be improved for future groups of students. Future work will also involve exploring how students use computation to understand diffusion as well as other concepts in our introductory physics course.

\section{ACKNOWLEDGMENTS}

This research was supported in part by the Michigan State University Graduate School and the MSU Lyman Briggs College Scholarship of Undergraduate Teaching and Learning Fellowship Program.
[7] E. F. Redish et al., "NEXUS/Physics: An interdisciplinary repurposing of physics for biologists," American Journal of Physics 82, 368-377 (2014).

[8] J. A. Morris and M. J. Gardner, "Statistics in Medicine: Calculating confidence intervals for relative risks (odds ratios) and standardised ratios and rates," British Medical Journal (Clinical Research Ed.) 296, 1313-1316 (1988).

[9] M. Cooper and M. Klymkowsky, "Chemistry, life, the universe, and everything: a new approach to general chemistry, and a model for curriculum reform," Journal of Chemical Education 90, 1116-1122 (2013).

[10] N. J. Tro, Chemistry: Structure and Properties (Pearson, New York, 2017).

[11] E. Brewe, "Modeling theory applied: Modeling Instruction in introductory physics," American Journal of Physics 76, 11551160 (2008). 